\title{
Seguimento farmacoterapêutico de pacientes em tratamento com talidomida em um centro especializado em hanseníase
}

\author{
Pharmacotherapeutic follow-up of patients treated with thalidomide \\ at a specialized leprosy center
}

\author{
Rafhael Lucas Holanda de Vasconcelos ${ }^{1,3}$, Webysten Ronny Pereira dos Santos ${ }^{1,3}$, Allyson Martins Lopes Sousa ${ }^{1,3}$, \\ Laynne Hellen de Carvalho Leal ${ }^{1,3}$, Luís Mario Rezende Júnior ${ }^{1}$, Jociane Alves da Silva Reis ${ }^{1}$, Dany Gabriel Sampa ${ }^{2}$, \\ Pablo Ricardo Barbosa Ferreira ${ }^{3}$, Éverton José Ferreira de Araújo ${ }^{1} \bowtie$ \\ 1 Departamento de Farmácia da Universidade Federal do Piauí (UFPI). Teresina, PI.
${ }^{2}$ Centro Maria Imaculada da Ação Social Arquidiocesana. Teresina, PI. \\ ${ }^{3}$ Farmácia Escola da UFPI. Espaço de Convivência Rosa dos Ventos. Teresina, PI.
}

\section{RESUMO}

OBJETIVOS: Avaliar a farmacoterapia de pacientes com reação hansênica tipo 2 em tratamento com talidomida em um centro filantrópico de atendimento especializado.

MÉTODOS: O estudo foi realizado no Centro Maria Imaculada de reabilitação de pacientes com hanseníase na cidade de Teresina, Piauí. Foram incluídos na pesquisa pacientes de ambos os sexos atendidos entre setembro e novembro de 2016. O Seguimento Farmacoterapêutico foi fundamentado no Método Dáder, na base eletrônica Drugdex System - Thomson Micromedex ${ }^{\circledR}$ - Interactions para análise de interações medicamentosas; na classificação de reações adversas a medicamentos de Rawlins e Thompson; e no teste de Morisky-Green para avaliar o nível de adesão terapêutica.

RESULTADOS: Foram acompanhados 11 pacientes, dos quais oito eram homens. Foram identificadas três interações medicamentosas, sendo duas classificadas em risco moderado e uma em menor risco. Foram identificados 23 resultados negativos associados ao medicamento, destacando-se insegurança não quantitativa e problemas de saúde não tratados. Além disso, 22 problemas relacionados com medicamentos foram identificados, sendo o mais frequente a ocorrência de reações adversas a medicamentos. Todas as reações adversas associadas a medicamentos foram classificadas como tipo A ou previsíveis. Quanto à adesão, seis entre os nove pacientes que responderam ao teste de Morisky-Green obtiveram alto grau de adesão. A educação em saúde correspondeu à intervenção farmacêutica preponderante, sendo aplicada a todos os pacientes.

CONCLUSÕES: Foram evidenciadas interações medicamentosas relevantes, resultados negativos associados ao medicamento e problemas associados a medicamentos. O grau de adesão ao tratamento com talidomida foi considerado alto. Foram necessárias intervenções farmacêuticas, sobretudo voltadas para ações de educação em saúde, o que ratifica a necessidade de acompanhamento constante desse grupo de pacientes.

DESCRITORES: farmacoterapia; atenção farmacêutica; efeitos colaterais e reações adversas relacionada a medicamentos; hanseníase; talidomida.

\section{ABSTRACT}

AIMS: Evaluate the pharmacotherapy of patients with type 2 leprosy reaction in treatment with thalidomide in a philanthropic center of specialized care at Teresina.

METHODS: The study was conducted at the Centro Maria Imaculada, for rehabilitation of patients with leprosy, in the city of Teresina, Piauí, Brazil. Patients of both sexes attended between september and november 2016 were included in the study. Pharmacotherapeutic follow-up was based on the Dáder Method, in the electronic base Drugdex System - Thomson Micromedex ${ }^{\mathbb{B}}$ - Interactions for analysis of drug interactions; in the classification of adverse drug reactions of Rawlins and Thompson; and the Morisky-Green test to evaluate the level of therapeutic adherence. RESULTS: Eleven patients were followed, of whom eight were male. Three drug interactions were identified, two of which were classified as moderate risk and one in lower risk. There were 23 negative results associated with medicines, mainly quantitative insecurity and untreated health problems. In addition, 22 drug-related problems were identified, with adverse drug reactions being the most frequent occurrence. All adverse drug reactions were classified as type A or predictable. Regarding adhesion, six patients among nine who responded to the Morisky-Green test obtained a high degree of adhesion. Health education corresponded to the preponderant pharmaceutical intervention, being applied to all patients.

CONCLUSIONS: Relevant drug interactions, negative results associated with medicines, and drug-related problems were identified. Degree of adherence to thalidomide treatment was considered high. Pharmaceutical interventions were necessary, mainly focused on health education actions, which ratifies the need for constant monitoring of this group of patients.

KEY WORDS: pharmacotherapy; pharmacotherapeutic care; side effects and drug-related adverse reactions; leprosy; thalidomide. 
Abreviaturas: ATC, Anatomical Therapeutic Chemical; OMS, Organização Mundial da Saúde; PRM, problemas relacionados a medicamentos; RAM, reações adversas a medicamentos; RDC, Resolução da Diretoria Colegiada; RNM, resultados negativos associados a medicamentos; SF, seguimento farmacoterapêutico.

\section{INTRODUÇÃO}

A hanseníase é uma doença infectocontagiosa de evolução crônica que se caracteriza, principalmente, por lesões cutâneas com diminuição da sensibilidade térmica, dolorosa e tátil, bem como acometimento de nervos periféricos, podendo resultar em deformidades e incapacidades físicas [1]. Considerada de alta infectividade, mas baixa patogenicidade, a manifestação clínica da hanseníase depende não só de suas características intrínsecas, mas também da relação agente-hospedeiro e do grau de endemicidade da região [2].

De acordo com dados da Organização Mundial da Saúde (OMS), até o primeiro trimestre do ano de 2014 o número de casos de hanseníase no mundo foi de 180.464 [3]. O Brasil é o segundo país em incidência dessa doença, sendo superado apenas pela Índia. No ano de 2014, foram registrados 31.064 novos casos de hanseníase no Brasil [4, 5]. Segundo estudo realizado pelo Ministério da Saúde entre 2011 e 2013, as áreas com maior risco da doença estão localizadas nas regiões Centro-Oeste, Norte e Nordeste [6].

O Piauí figura entre os cinco estados de maior número de casos de hanseníase na região Nordeste. De acordo com dados do Ministério da Saúde, em 2014 foram registrados 1.038 novos casos da doença em todo o estado, sendo que deste número, 76 foram acometimentos em menores de 15 anos [6]. Dados da Secretaria de Estado de Saúde do Piauí, referentes ao ano de 2015, mostram que o registro de 901 novos casos de hanseníase. Em 2016, o estado apresentou 775 novos casos da doença com obtenção de cura em $78,8 \%$ dos casos [7].

A escolha da terapêutica é fundamentada na classificação operacional elaborada pela OMS, a qual foi baseada em critérios clínicos. A forma paucibacilar, caracterizada por cinco lesões cutâneas e/ou um tronco nervoso acometido, tem tratamento associado ao uso dos fármacos dapsona e rifampicina, com possibilidades de esquemas alternativos com ofloxacina e minociclina. Já forma multibacilar, que agrupa os casos em que se observa mais de cinco lesões cutâneas e/ou mais de um tronco nervoso acometido, tem esquema terapêutico com rifampicina, dapsona e clofazimina. No que concerne às reações hansênicas, o tratamento consiste fundamentalmente no uso do glicocorticoide prednisona para as reações do tipo 1 e do imunomodulador talidomida para reações do tipo 2 [8-10].

Paralelamente ao tratamento sujeito à prescrição e ao acompanhamento médico, a realização do seguimento farmacoterapêutico (SF) pelo farmacêutico contribui para a obtenção de melhores resultados na farmacoterapia instituída e na qualidade de vida dos pacientes [11]. A participação de uma equipe de saúde multiprofissional, como provedora de atenção ao paciente, é apontada pela OMS como estratégia de melhoria dos serviços de saúde [12].

A prática da atenção farmacêutica em pacientes com hanseníase no Brasil desenvolveu-se consideravelmente nos últimos anos. Entidades como o Conselho Federal de Farmácia e o Ministério da Saúde têm delegado maiores responsabilidades ao profissional farmacêutico que, inserido no processo de cuidado básico, tem o dever de divulgar informações sobre a doença, além de encaminhar possíveis novos casos para o adequado exame clínico. Em relação ao uso de talidomida, a Resolução da Diretoria Colegiada (RDC) no 11 de 22 de março de 2011 da Agência Nacional de Vigilância Sanitária, preconiza que é indispensável a assistência farmacêutica efetiva com orientação e monitoramento do uso do medicamento. Os pacientes em uso dessa medicação devem ter uma dispensação especializada e diferenciada que garanta a compreensão sobre a doença e o tratamento. Os serviços de saúde que assistem aos usuários da talidomida devem verificar todos os medicamentos utilizados pelo paciente, conciliar o seu uso e monitorar a adesão ao tratamento $[13,14]$.

O presente estudo visou avaliar a farmacoterapia de pacientes com hanseníase em estado reacional tipo 2 em tratamento com talidomida em um centro filantrópico de atendimento especializado de Teresina, Piauí, Brasil.

\section{MÉTODOS}

Foi aplicado um programa de SF a pacientes portadores de hanseníase em estado reacional tipo 2, que utilizavam o medicamento talidomida. O estudo foi realizado entre setembro e novembro de 2016, no Centro Maria Imaculada de reabilitação de pacientes com hanseníase, na cidade de Teresina, Piauí, uma instituição filantrópica mantida pela Ação Social Arquidiocesana e por voluntariado. Foram incluídos na pesquisa pacientes de ambos os sexos, residentes em Teresina que, independentemente da idade, 
apresentavam hanseníase em estado reacional do tipo 2 em vigência de terapia com talidomida há pelo menos três meses.

Este estudo seguiu os preceitos éticos estabelecidos na Resolução 466/12 do Conselho Nacional de Saúde, preservando a identidade dos participantes, e foi aprovado pelo Comitê de Ética em Pesquisa da Universidade Federal do Piauí, sendo registrado sob o Certificado de Apresentação para Apreciação Ética no 56010316.3.0000.5214 e Parecer Consubstanciado $\mathrm{n}$ - 1.618.502. Todos os participantes que concordaram em participar assinaram o Termo de Consentimento Livre e Esclarecido.

O SF foi fundamentado no Método Dáder, desenvolvido pelo Grupo de Investigação em Atenção Farmacêutica da Universidade de Granada, Espanha, com as classificações acordadas em 2007 no Terceiro Consenso de Granada sobre resultados negativos associados ao medicamento (RNM) e problemas relacionados com medicamentos (PRM) [14]. Foram realizadas sucessivas entrevistas, empregando a metodologia fundamentada no método Dáder, conforme as seguintes etapas: $1 \underline{\text { a }}$ etapa) oferta do serviço e agendamento da primeira entrevista; $2^{\underline{a}}$ etapa) primeira entrevista com análise da situação do paciente e apresentação da sacola de medicamentos e exames laboratoriais realizados nos últimos três meses; 3 a etapa) fase de estudo em que se realizou a avaliação da farmacoterapia e de interações medicamentosas, com auxílio da base Drugdex System - Thomson Micromedex ${ }^{\circledR}$ - Interactions, classificação Anatomical Therapeutic Chemical (ATC) da OMS e da literatura científica especializada; 4aㅡ etapa) fase de avaliação de suspeitas de PRM e RNM; 5a etapa) segunda entrevista para realização de intervenções farmacêuticas; e 6 ${ }^{\text {a }}$ etapa) terceira entrevista para acompanhamento das intervenções realizadas. Além do exposto, durante o andamento do SF foi ofertada aos pacientes a realização de aferição da glicemia capilar e da pressão arterial sistêmica.

As interações medicamentosas foram identificadas e classificadas pela base Drugdex System - Thomson Micromedex ${ }^{\circledR}$ - Interactions, em três categorias: a) risco maior, quando a interação pode ter risco de morte e/ou requer intervenção médica e/ou requer uma alteração na terapia; b) risco moderado, quando a interação pode resultar em uma exacerbação da condição do paciente; c) risco menor, quando a interação pode ter efeitos clínicos limitados, incluindo o aumento na frequência ou gravidade dos efeitos colaterais, porém sem requerer maiores alterações na terapia $[15,16]$. Os medicamentos coletados durante o SF foram analisados conforme o segundo nível de classificação ATC da OMS, que categoriza os princípios ativos em classes terapêuticas por meio de um sistema de códigos de dois dígitos [17].

Os RNM foram classificados quanto à necessidade, efetividade e segurança. Quanto à necessidade, avaliou-se se o paciente tinha um problema de saúde por não utilizar a medicação que necessita, ou por utilizar um medicamento que não necessita; quanto à efetividade, se o paciente tinha um problema de saúde por uma inefetividade não quantitativa ou quantitativa da medicação; quanto à segurança, se o doente tinha um problema de saúde por uma insegurança não quantitativa ou quantitativa de um medicamento [14].

Os PRM reuniram as circunstâncias que poderiam causar ou resultar em RNM. Essas situações foram enquadradas nas seguintes ocorrências evidenciadas no SF: administração errônea do medicamento; conservação inadequada; presença de contraindicações; dose e/ou duração inadequada de terapia; não adesão medicamentosa; problema de saúde insuficientemente tratado; probabilidade de ocorrência de reações adversas a medicamentos (RAM) e outros problemas relacionados a medicamentos [14]. As RAM foram classificadas com base nos critérios propostos por Rawlins e Thompson [18], em reações do tipo A ou previsíveis, e reações do tipo B ou imprevisíveis [19].

O grau de adesão dos pacientes foi avaliado na primeira entrevista mediante aplicação única do formulário Medida de Adesão aos Tratamentos, proposto por Morisky e Green e modificado por Sewicht $[13,19,20]$. Essa medida é baseada nas respostas a quatro perguntas: $\left.1^{\underline{a}}\right)$ " $\mathrm{O}(\mathrm{A})$ senhor(a), às vezes, esquece de tomar seus remédios?"; 2므) “O(A) senhor(a), às vezes, se descuida quanto ao horário de tomar seus remédios?"; 3a) "Quando o(a) senhor(a) se sente bem, às vezes deixa de tomar seus remédios?"; 4ㅁ) "Quando o(a) senhor(a) se sente mal com seus remédios, às vezes deixa de tomá-los?" [20]. Classificou-se como alta adesão, o paciente que teve todas as respostas negativas e baixa adesão quando pelo menos uma resposta foi positiva.

\section{RESULTADOS}

Aceitaram participar do estudo 11 pacientes, sendo oito do gênero masculino. A Tabela 1 apresenta a distribuição dos medicamentos utilizados pelos pacientes conforme o segundo nível de classificação ATC, a qual demonstra os subgrupos terapêuticos e a distribuição de 16 princípios ativos presentes em medicamentos que estavam sendo 
utilizados concomitantemente com a talidomida pelos participantes da pesquisa. Destaca-se a maior frequência dos subgrupos M01 (anti-inflamatórios e antirreumáticos), A02 (antiácidos, antiulcerosos e antiflatulentos) e L04 (corticoides). Foi obtida média de 3,18 medicamentos por paciente.

Foram encontradas três possíveis interações medicamentosas entre os pacientes acompanhados. Entre estas, duas interações apresentaram distribuição para risco moderado e uma para risco menor. As interações consideradas de risco moderado consistiram na existente entre o diurético hidroclorotiazida e o gastroprotetor omeprazol, e a de menor risco foi relacionada ao uso associado de prednisona e hidroclorotiazida.

Quatro participantes relataram a ingestão regular de alimentos gordurosos em horários distintos ao de administração do medicamento talidomida. Quatro pacientes apresentaram exames laboratoriais, entre os quais dois possuíam perfil lipídico alterado, com valor médio de colesterol total de $214 \mathrm{mg} / \mathrm{dL}$. Uma paciente encontrava-se em idade fértil, tinha vida sexual ativa e não utilizava nenhum método contraceptivo oral, além de não possuir o hábito de realizar teste de gravidez mensalmente. Dois pacientes apresentavam diabetes melito tipo 2 e um apresentava hipertensão arterial sistêmica, todos diagnosticados antes da hanseníase.

Foram identificados 23 RNM (Tabela 2) e 22 PRM (Tabela 3) como suas causas. As RAM encontradas no presente estudo foram todas classificadas como previsíveis ou do tipo A. Nove pacientes concordaram em responder ao questionário de adesão à talidomida. Entre eles, seis apresentaram alto grau de adesão. Os outros três relataram já ter esquecido de tomar o medicamento e/ou se descuidar do horário de administração, sendo, portanto, classificados como pacientes de baixa adesão.

Foram planejadas 16 intervenções farmacêuticas, das quais 12 corresponderam à educação em saúde; três foram sugestões escritas ao prescritor de retirada de medicamentos e uma correspondia a orientações quanto ao modo de uso e administração.

As sugestões ao prescritor de retirada de medicamentos foram feitas verbalmente ao paciente $\mathrm{e}$ de forma escrita, a fim de que o mesmo as levasse ao prescritor. Ademais, foi sugerida a utilização de esquemas didáticos para contornar as dificuldades de identificação dos medicamentos ou do horário de administração. Para isso, os medicamentos foram marcados com adesivos de cores distintas com significado específico.
As intervenções farmacêuticas foram bem aceitas pelos pacientes. Até a conclusão da terceira entrevista, todos os pacientes orientados suspenderam o uso de medicamentos cujo uso havia sido associado à prática da automedicação e/ou haviam agendado consultas com especialistas da área da saúde para uma avaliação mais criteriosa das suas terapias medicamentosas e hábitos de vida.

Tabela 1. Subgrupos terapêuticos e princípios ativos dos medicamentos utilizados pelos 11 pacientes participantes da pesquisa, de acordo com o segundo nível de classificação Anatomical Therapeutic Chemical. Teresina, Piauí, 2017.

\begin{tabular}{llc}
\hline Subgrupo & Classe terapêutica & $\begin{array}{c}\text { Princípios ativos } \\
\text { n (\%) }\end{array}$ \\
\hline M01 & Anti-inflamatórios/ antirreumáticos & $4(25)$ \\
A02 & Antiácidos/ antiulcerosos/ antiflatulentos & $4(25)$ \\
L04 & Corticosteroides & $3(18,7)$ \\
A10 & Hipoglicemiantes & $2(12,4)$ \\
G03 & Hormônios sexuais & $1(6,3)$ \\
C03 & Diuréticos & $1(6,3)$ \\
SC & Sem classificação & $1(6,3)$ \\
Total & & $16(100)$ \\
\hline
\end{tabular}

Tabela 2. Resultados negativos associados a medicamentos identificados durante o seguimento farmacoterapêutico de 11 pacientes com reação hansênica tipo 2 em uso de talidomida. Teresina, Piauí, 2017.

\begin{tabular}{|c|c|}
\hline RNM observados & n (\%) \\
\hline Insegurança não quantitativa ao uso de medicamentos & $8(34,8)$ \\
\hline $\begin{array}{l}\text { Resultados negativos associados a problemas de saúde } \\
\text { não tratados }\end{array}$ & $6(26)$ \\
\hline Problemas de saúde associados a não necessidade & $5(21,8)$ \\
\hline Inefetividade quantitativa & $4(17,4)$ \\
\hline Total & $23(100)$ \\
\hline
\end{tabular}

RNM, resultados negativos associados a medicamentos.

Tabela 3. Problemas relacionados a medicamentos identificados durante o seguimento farmacoterapêutico de 11 pacientes com reação hansênica tipo 2 em uso de talidomida. Teresina, Piauí, 2017.

\begin{tabular}{lc}
\hline PRM observados & n (\%) \\
\hline Problema de saúde insuficientemente tratado & $5(22,8)$ \\
Probabilidade de reações adversas a medicamentos & $5(22,8)$ \\
Dose e/ou duração inadequada da terapia & $4(18,2)$ \\
Não adesão medicamentosa & $3(13,6)$ \\
Administração errônea do medicamento & $2(9,1)$ \\
Conservação inadequada do medicamento & $1(4,5)$ \\
Presença de contraindicações & $1(4,5)$ \\
Outros problemas de saúde que afetam o tratamento & $1(4,5)$ \\
Total & $22(100)$ \\
\hline
\end{tabular}

PRM, problemas relacionados a medicamentos. 


\section{DISCUSSÃO}

Nesta amostra de pacientes hansênicos houve predominância do gênero masculino. Foram evidenciadas três interações medicamentosas e risco de interação medicamento-alimento. Houve associação da talidomida predominantemente a medicamentos dos subgrupos ATC M01, A02 e L04. Foram identificados 23 RNM, 22 PRM e RAM previsíveis. Quanto à adesão, seis entre nove pacientes obtiveram alto grau de adesão. Entretanto, foram necessárias intervenções farmacêuticas, sobretudo de educação em saúde.

De acordo com a Sociedade Brasileira de Dermatologia, a hanseníase atinge principalmente a população economicamente ativa, na faixa etária entre 13 e 50 anos. Embora acometa ambos os gêneros, observa-se predominância do gênero masculino, o que foi observado na amostra do presente estudo. Pesquisas realizadas por Batista et al. [21] e Duarte et al. [22] corroboram com o fato da doença ser mais incidente em indivíduos do gênero masculino.

Os pacientes portadores de doenças crônicas possuem no decurso do seu tratamento o agravante da prática da polifarmácia, o que predispõe a interações medicamentosas e RAM, elevando o risco associado às terapias medicamentosas. $\mathrm{Na}$ presente amostra, os anti-inflamatórios destacaram-se entre os subgrupos terapêuticos mais utilizados juntamente com a talidomida. Esse subgrupo merece a atenção das equipes de saúde em virtude dos riscos associados principalmente a lesões gástricas, assim como o grupo composto por antiácidos, antiulcerosos e antiflatulentos, que podem ser classificados como medicamentos isentos de prescrição médica. Os medicamentos isentos de prescrição têm seu uso muito associado com a prática da automedicação, o que reforça a necessidade da atuação do profissional farmacêutico [22, 23].

Deve-se destacar ainda o uso concomitante de glicocorticoides, pois três pacientes do estudo faziam uso dessa classe de medicamentos juntamente com a talidomida. Um paciente utilizava concomitantemente alendronato de sódio, carbonato de cálcio e vitamina $\mathrm{D}$, uma vez que o uso crônico de glicocorticoides é reconhecidamente um fator de risco para osteoporose e fraturas. A literatura científica relata que os glicocorticoides diminuem a massa óssea e assim aumentam o risco de fraturas, particularmente fraturas das costelas, coluna vertebral e antebraço [24].

A análise das interações medicamentosas potenciais entre os participantes revelou interação com risco moderado entre a hidroclorotiazida e o omeprazol. Segundo Thongon e Krishnamra [25], o omeprazol tem um efeito inibidor sobre a absorção passiva de magnésio, portanto, seu uso concomitante a um diurético tiazídico pode resultar em hipomagnesemia. Outra possível interação foi verificada entre a prednisona e a hidroclorotiazida, classificada como de risco menor pela base Micromedex $^{\circledR}$. Esta interação pode resultar em hipocalemia e subsequentes arritmias cardíacas, consoante estudos realizados por Formighieri [26], haja vista que a hidroclorotiazida promove o aumento da excreção de potássio.

Houve ainda indícios de interação medicamentoalimento, considerando que a administração de talidomida concomitantemente a alimentos gordurosos pode maximizar o tempo requerido para atingir o pico de concentração plasmática. Esta informação tem suma importância se observado que quatro pacientes, mais de um terço dos participantes, relataram a ingestão regular de alimentos gordurosos, apesar de não a realizarem em horários próximos à administração da talidomida. [12, 15, 25-29].

Apenas quatro pacientes realizavam exames laboratoriais periódicos, o que demonstra certo descuido ou desconhecimento quanto a essa questão. Quanto aos exames laboratoriais, destacou-se o lipidograma completo, apresentado por todos os quatro pacientes. Dentre estes, dois encontravam-se com valores de colesterol total acima do valor desejável de $200 \mathrm{mg} / \mathrm{dL}$, fato associável à questão da dieta hipercalórica [30].

Em relação ao uso de talidomida por mulheres em idade fértil, é previsto pela lei no 10.651 de 16 de abril de 2003 e pela RDC no 11 de 22 de março de 2011 da Agência Nacional de Vigilância Sanitária que a prescrição seja feita em formulário numerado e especial, sendo obrigatória a assinatura do termo de consentimento livre e esclarecido pela paciente e pelo prescritor. Entretanto, identificou-se que uma paciente descumpria a legislação sanitária, uma vez que não realizava exames de $\beta$-HCG periódicos e nem utilizava método anticoncepcional regularmente, o que demonstra desconhecimento da alta magnitude do risco de teratogenicidade associado à isotretinoína [31, 32].

Quanto aos RNM identificados, situações de insegurança não quantitativa quanto ao uso de medicamentos e os resultados negativos associados a problemas de saúde não tratados apresentaram maior frequência. Ressalta-se o RNM decorrente de problema de saúde não tratado associado ao risco dos pacientes desenvolverem osteoporose induzida por corticosteroides, já que a prednisona é utilizada para tratar casos de reações hansênicas, sendo comum seu uso prolongado em pacientes com hanseníase [33-34]. 
Dentre os RNM, deve-se ressaltar o medicamento não necessário e a inefetividade quantitativa, que nos casos acompanhados decorreram, respectivamente, da utilização de anti-inflamatórios não esteroidais, e do esquecimento dos horários de administração ou até mesmo abandono da terapêutica medicamentosa $[35,36]$.

Dentre os possíveis PRM causadores dos RNM identificados, houve destaque para a probabilidade de ocorrência de RAM, as quais no presente estudo compreenderam a reações do tipo A ou previsíveis. Essas RAM corresponderam à reação observada nos pacientes que utilizavam regularmente corticosteroides e que apresentavam queixas de dores musculares e articulares [36-38].

Quanto à adesão terapêutica à talidomida, a maioria dos pacientes que aceitaram responder ao questionário (seis entre os nove) foram considerados com alto grau de adesão, o que assinala preocupação e cuidado com a saúde. Em contrapartida, houve dois pacientes que se negaram a responder ao questionário de adesão e três foram considerados pouco aderentes, o que enaltece a importância do acompanhamento constante dos pacientes com a reação hansênica tipo 2. Não há no meio científico um método considerado padrão ouro para avaliação da adesão terapêutica. Além disso, ensaios dependentes de questionários estão sujeitos a fatores subjetivos, tais como a negativa dos pacientes $[37,39]$. Entretanto sabe-se que o conhecimento dos pacientes sobre os seus problemas de saúde e sobre a importância do tratamento contribui para maior adesão terapêutica e, consequentemente, para alcançar o objetivo do tratamento [26, 40, 41].

Como consequência dos resultados obtidos na avaliação realizada, a educação em saúde foi a intervenção farmacêutica mais frequente, sendo aplicada a todos os pacientes participantes da pesquisa. As intervenções farmacêuticas tiveram boa receptividade por parte dos pacientes, ratificando com a importância do profissional farmacêutico no cuidado ao paciente sob terapia medicamentosa.

\section{NOTAS}

\section{Agradecimentos}

Ao Centro de Apoio Maria Imaculada e à Farmácia Escola da Universidade Federal do Piauí, pelo incentivo à realização da pesquisa e disponibilidade de infraestrutura e pessoal capacitado para o desenvolvimento do seguimento farmacoterapêutico proposto.

\section{Apoio financeiro}

Este estudo não recebeu apoio financeiro de fontes externas.

Declaração de conflito de interesses

Os autores declaram não haver conflitos de interesses relevantes ao conteúdo deste estudo, informam ter tido acesso a todos os dados obtidos e assumem completa responsabilidade pela integridade dos resultados.

\section{REFERÊNCIAS}

1. Crespo MJI, Gonçalves A, Padovani CR. Hanseníase: pauci e multibacilares estão sendo diferentes? Medicina (Ribeirão Preto. Online). 2014;47(1):43-50.

2. Brasil. Ministério da Saúde. Secretaria de Vigilância em Saúde. Departamento de Vigilância Epidemiológica. Guia de Vigilância Epidemiológica. 7̣a edição. Brasília: Ministério da Saúde; 2009. 816p.

3. Organização Mundial da Saúde. Leprosy Today [Internet]. Geneva; 2015. [cited 2016 october 18]. Available from: http:// www.who.int/lep/en/

4. World Health Organization. Global leprosy situation, 2012. Weekly Epidemiological Record (WER). Genebra. 2012;87(34):317-28.

5. Brasil. Ministério da Saúde. Secretaria de Vigilância em Saúde. Departamento de Vigilância Epidemiológica. Orientações para uso: corticosteroides em hanseníase. Brasília: Ministério da Saúde; 2010. 52p.

6. Brasil. Ministério da Saúde. Secretaria de Ciência, Tecnologia e Insumos Estratégicos. Departamento de Assistência Farmacêutica e Insumos Estratégicos. Formulário Terapêutico Nacional 2010: Rename 2010. 2a edição. Brasília: Ministério da Saúde; 2010. 1135p.

7. Piauí. Secretaria de Estado de Saúde do Piauí (SESAPI). Sistema de Informação de Agravos de Notificação. Boletim Epidemiológico do Estado do Piauí - Hanseníase. Teresina: SESAPI; 2016. 6p.

8. Brasil. Ministério da Saúde. Secretaria de Políticas de Saúde. Departamento de Atenção Básica. Guia para o Controle da hanseníase. Brasília: Ministério da Saúde; 2002.

9. Ura S. Tratamento e controle das reações hansênicas. Hansenol Int. 2007;32(1):67- 70.

10. Lastória JC, Abreu MAMM. Hanseníase: diagnóstico e tratamento. Diagn Tratamento. 2012;17(4):173-9.

11. Hepler CD, Strand LM. Opportunities and responsibilities in the pharmaceutical care. Am J Hosp Pharm. 1990;47:533-43. 
12. Brasil. Ministério da Saúde. Secretaria de Políticas de Saúde. Departamento de Atenção Básica. Guia para o Controle da Hanseníase. Brasília: Ministério da Saúde; 2002. 89p.

13. Brasil. Ministério da Saúde. Secretaria de Vigilância em Saúde. Departamento de Vigilância das Doenças Transmissíveis. Talidomida: orientação para o uso controlado. Brasília: Ministério da Saúde; 2014. 100p.

14. Dáder MJF, Hernández DS, Castro MMS. Método Dáder: Manual de Seguimento Farmacoterapêutico. 3a edição. Alfenas: Editora da Universidade Federal de Alfenas; 2014. 128 p.

15. Santos AC, Pereira DA, Silva, AO, Lopes LC. Seguimento farmacoterapêutico em pacientes com tuberculose pulmonar através da Metodologia Dáder. Rev Cienc Farm Basic Aplic. 2006;27(3):269-73.

16. Silva JMGC, Grellet MN, Liermann LC, Pastore CA, Vohlbrecht MBC, Bender E. Perfil nutricional do grupo de HIPERDIA da unidade básica de saúde do bairro Dunas, Pelotas-RS. In: 7. ENPOS, 2010, Pelotas. Pelotas: UFPEL; 2010.

17. Pádua CAM. Aspectos conceituais e abordagens metodológicas em farmacoepidemiologia. In: Acúrcio FA. Medicamentos: Políticas, Assistência Farmacêutica, Farmacoepidemiologia e Farmacoeconomia. Belo Horizonte: Ed. Coopmed; 2013. p. 75-112.

18. Rawlins MD, Thompson JW. Pathogenesis of adverse drug reactions. In. Davies DM, editor. Teextbook of Adverse Drug Reactions. Oxford: Oxford University Press; 1977. p.10-31.

19. Laporte JR, Capellà D. Mecanismos de producción y diagnóstico clínico de los efectos indeseables producidos por medicamento. In: Laporte JR, Tognoni G, editores. Principios de Epidemiología del Medicamento. $2^{\mathrm{a}}$ ed. Barcelona: Masson; 1993. p. 99-100.

20. Morisky DE, Green LW, Levine DM. Concurrent and predictive validity of a self-reported measure of medication adherence. Med Care. 1986; 24(1):67-74. https://doi.org/10.1097/00005650-198601000-00007

21. Batista ES et al. Perfil sócio-demográfico e clínico-epidemiológico dos pacientes diagnosticados com hanseníase em Campos dos Goytacazes, RJ. Rev Bras Clin Med. 2011;9(2):101-6.

22. Duarte MTC, Ayres JA, Simonetti JP. Perfil socioeconômico e demográfico de portadores de hanseníase atendidos em consulta de enfermagem. Rev Latino-Am. Enfermagem. 2007;15:774-9. https://doi.org/10.1590/S0104-11692007000700010

23. Hammes JA, Pfuetzenreiter F, Silveira F, Koenig A, Westphal GA. Prevalência de potenciais interações medicamentosas droga-droga em unidades de terapia intensiva. Rev Bras Ter Intensiva. 2008;20(4):349-54. https://doi.org/10.1590/S0103507 X2008000400006

24. Kanis JA, Stevenson M, McCloskey EV, Davis S, Lloyd-Jones M. Glucocorticoid-induced osteoporosis: a systematic review and cost-utility analysis. Health Technol Assess. 2007;11(7):3-4. https://doi.org/10.3310/hta11070

25. Thongon N, Krishnamra N. Apical acidity decreases inhibitory effect of omeprazole on Mg2+ absorption and claudin-7 and -12 expression in Caco-2 monolayers. Exp Mol Med. 2012;44(11):684-93. https://doi.org/10.3858/emm.2012.44.11.077

26. Formighierei RV. Interações relatadas para medicamentos que compõem a lista da Farmácia Popular do Brasil. Porto Alegre: Universidade Federal do Rio Grande do Sul; 2008. 96p.

27. Garcia BP, Myamoto CA. Talidomida - I Propriedades Farmacológicas e Toxicológicas. Rev Conex Eletrônica. 2016;13(1):425-34.

28. Flores L, Segura C, Quesada MS, Hall V. Seguimiento Farmacoterapéutico con el Método Dáder en un grupo de pacientes con hipertensión arterial. Seguim Farmacoterap. 2005;3(3):154-7.

29. Marques LA, Galduróz JC, Fernandes MR, Oliveira CC, Beijo LA, Noto AR. Assessment of the effectiveness of pharmacotherapy follow-up in patients treated for depression. J Managed Care Pharma (JMCP). 2013;19(3):218-27. https://doi.org/10.18553/jmcp.2013.19.3.218

30. Sociedade Brasileira de Cardiologia. V Diretriz Brasileira de Dislipidemias e Prevenção da Aterosclerose. Arq Bras Cardiol. 2013;101(4)Supl 1. http://dx.doi.org/10.5935/abc.2013S010

31. Brasil. Agência Nacional de Vigilância Sanitária. Resolução RDC no 11, de 22 de março de 2011. Dispõe sobre o controle da substância Talidomida e do medicamento que a contenha. Diário Oficial da União, 24 de março de 2011.

32. Brasil. Agência Nacional de Vigilância Sanitária. Lei no 10.651, 16 de abril de 2003. Dispõe sobre o controle do uso da talidomida. Diário Oficial da União, 17 de abril de 2003. Seção 1. p. 25.

33. Brasil. Ministério Da Saúde. Portaria no 3.125, de 7 de outubro de 2010. Aprova as Diretrizes para a vigilância, a atenção e o controle da hanseníase. Diário Oficial da União, no 198, 15 de outubro de 2010. Seção 1. p. 55.

34. Reis BC. Seguimento farmacoterapêutico com usuários de Talidomida atendidos pelo hospital universitário de Brasília (HUB). Ceilândia: Universidade de Brasília; 2013. 54p.

35. Brenol JCT, Xavier RM, Marasca J. Antiinflamatórios não hormonais convencionais. Rev Bras Med. 2000;57:33-40.

36. Renovato RD. Implementação da atenção farmacêutica para pacientes com hipertensão e desordens cardiovasculares. Infarma. 2002;14(7/8):52-6.

37. Souza TRCL, Silva AS, Leal LB, Santana DP. Método Dáder de Seguimento Farmacoterapêutico. 3ํe edição (2007): Um estudo piloto. Rev Cienc Farma Básica Apl. 2009;30(1):90-4. 
38. Chemello C, Aguilera M, Calleja-Hernández MA, Fausa MJ. Efecto del seguimiento farmacoterapéutico en pacientes con hiperparatiroidismo secundario tratados con cinacalcet. Farm Hosp. 2012;36(5):321-7. https://doi.org/10.1016/j. farma.2011.03.008

39. Magalhães SMS, Carvalho WS. Reações adversas a medicamentos. In: Gomes MJVM, Morreira AM, editores. Ciências farmacêuticas: uma abordagem em farmácia hospitalar. São Paulo: Atheneu; 2001. p. 125-45.

40. Ben AJ, Neumann CR, Mengue SS. Teste de Morisky-Green e Brief Medication Questionnaire para avaliar adesão a medicamentos. Rev Saúde Pública. 2012;46(2):279-89. https://doi.org/10.1590/S0034-89102012005000013

41. Amarante LC, Shoji LS, Beijo LA, Lourenço EB, Marques LAM. A influência do acompanhamento farmacoterapêutico na adesão à terapia anti-hipertensiva e no grau de satisfação do paciente. Rev Ciênc Farm Básica Apl. 2010;31(3):209-15. 